\title{
BMJ Open Protocol for a qualitative study exploring the perception of need, importance and acceptability of a digital diabetes prevention intervention for women with gestational diabetes mellitus during and after pregnancy in Malaysia (Explore-MYGODDESS)
}

To cite: Sobri NHM, Ismail IZ, Hassan F, et al. Protocol for a qualitative study exploring the perception of need, importance and acceptability of a digital diabetes prevention intervention for women with gestational diabetes mellitus during and after pregnancy in Malaysia (ExploreMYGODDESS). BMJ Open 2021;11:e044878. doi:10.1136/ bmjopen-2020-044878

- Prepublication history and additional supplemental material for this paper are available online. To view these files, please visit the journal online. (http://dx.doi.org/10.1136/ bmjopen-2020-044878).

Received 16 September 2020 Accepted 06 August 2021

D) Check for updates

(C) Author(s) (or their employer(s)) 2021. Re-use permitted under CC BY-NC. No commercial re-use. See rights and permissions. Published by BMJ.

For numbered affiliations see end of article.

Correspondence to Dr Boon How Chew; chewboonhow@upm.edu.my

\section{ABSTRACT}

Introduction Women who develop gestational diabetes mellitus (GDM) have an increased risk of developing type 2 diabetes, and to reduce this risk the women have to adopt healthy behaviour changes. Although previous studies have explored the challenges and facilitators to initiate behaviour change among women with GDM, there is limited data from Malaysian women. Thus, this study will explore the factors affecting the uptake of healthy behaviour changes and the use of digital technology among women and their healthcare providers (HCPs) to support healthy behaviour changes in women with GDM. Methods and analysis The study will be modelled according to the Capability, Opportunity, Motivation and Behaviour and Behaviour Change Wheel techniques, and use the DoTTI framework to identify needs, solutions and testing of a preliminary mobile app, respectively. In phase 1 (design and development), a focus group discussion (FGDs) of 5-8 individuals will be conducted with an estimated 60 women with GDM and 40 HCPs (doctors, dietitians and nurses). Synthesised data from the FGDs will then be combined with content from an expert committee to inform the development of the mobile app. In phase 2 (testing of early iterations), a preview of the mobile app will undergo alpha testing among the team members and the app developers, and beta testing among 30 women with GDM or with a history of GDM, and 15 HCPs using semi-structured interviews. The outcome will enable us to optimise an intervention using the mobile app as a diabetes prevention intervention which will then be evaluated in a randomised controlled trial.

Ethics and dissemination The project has been approved by the Malaysia Research Ethics Committee. Informed consent will be obtained from all participants. Outcomes will
Strengths and limitations of this study

- A new digital diabetes prevention intervention tool (mobile application) will be fully contextualised to the local settings and expectations modelled according to the Capability, Opportunity, Motivation and Behaviour and Behaviour Change Wheel techniques.

- The DoTTI framework approach for web-based information tools and software is adopted to pilot the evaluation of the needs assessment, content development, app interface designing as well as alpha and beta testing.

- Respondents of heterogeneous sociodemographic and professional backgrounds from multiple public healthcare facilities are recruited via purposive sampling.

- Respondents are largely recruited from urban and more developed regions in Malaysia where the study is centred and this may cause a lack of representation of women from lower-income and rural settings (we will monitor this in the recruitment process).

- Data collected through online meeting platforms could affect data quality as on online platform limits the observation of the non-verbal aspects of both the interviewer and note taker during the interviews.

be presented at both local and international conferences and submitted for publications in peer-reviewed journals.

\section{INTRODUCTION}

The International Diabetes Federation states that one in six live births are affected 
by gestational diabetes mellitus (GDM), and majority of the cases are from low-income and middle-income countries. ${ }^{1}$ GDM in South East Asia has an estimated prevalence rate ranging from $10.3 \%$ to $28.5 \% .^{1-3}$ Generally, GDM is associated with numerous complications that can affect mothers. For example, women with GDM are more likely to have pre-eclampsia and three times more likely to deliver via caesarean section. ${ }^{45}$ Besides, these women also have a sevenfold increased risk of developing type 2 diabetes (T2D) within 5-15 years of postdelivery, ${ }^{6} 7$ and over half of the women will develop pre-diabetes. ${ }^{8}$

The management of GDM aims for optimal glycaemic control and weight gain during pregnancy in an effort to minimise the risk of women from developing T2D. This requires lifestyle modification which consists of dietary modification, regular exercise and blood glucose monitoring with or without metformin or insulin. ${ }^{7-14}$ This intensive management ends after delivery, whereas the risk of developing T2D remains. ${ }^{14}$ As a result, women with a history of GDM are regarded as high-risk individuals to develop T2D in the future. Therefore, there is a need for postpregnancy diabetes prevention interventions (DPIs)..$^{15}$ DPIs are defined as structured lifestyle modifications that prevent or delay the onset of T2D. ${ }^{16}$ In order to ensure optimal weight loss, good dietary routine and physical activity habits are introduced during the management of GDM are continued. ${ }^{16}{ }^{17}$ Randomised controlled trials have proven the effectiveness of DPIs in preventing or delaying the onset of T2D among women with a history of GDM by applying lifestyle modifications and weight $\operatorname{loss}^{17}$ which are found to be as effective as taking metformin. ${ }^{1617}$

However, women with GDM have described a range of challenges to uptake DPIs ${ }^{18-23}$ which include lack of information and peer support, negative emotional experience of a medicalised pregnancy and guilt. They also fear that they are to blame for being diagnosed with GDM which can potentially harm their baby. ${ }^{20-24}$ Lack of psychological support from healthcare providers (HCPs) has also been reported by women with newly diagnosed GDM as one of the challenges to uptake DPIs. These women also reported that they have received judgemental and critical comments about their condition from HCPs when they were in the process of making adjustments to new life routines, implementing diabetes self-care and making lifestyle changes. ${ }^{22-25}$

On the other hand, HCPs have expressed difficulties in managing women with GDM. These include a lack of medical knowledge about the biology of GDM, limited evidence for the most effective lifestyle interventions and lack of communication skills, self-help resources and guidance to share with patients. ${ }^{25-28}$

The challenges reported from face-to-face DPIs procedures have presented an opportunity to develop other approaches to overcome the issues. One approach is by using a digitalised DPIs which is usually introduced for internet, mobile phones or devices, telemedicine and technology that uses decision support techniques. ${ }^{29} \mathrm{~A}$ mobile app can combine decision support techniques into a cellular phone.$^{30} \mathrm{~A}$ review of 12 articles related to usage of mobile apps among women with GDM found that usage of such apps is beneficial in increasing the confidence levels in both the patients and clinicians. ${ }^{31-33}$ Furthermore, the app can be a platform for sending medication and diet information, obtaining feedback from clinicians $^{33}$ and sharing blood glucose self-monitoring. ${ }^{34-36} \mathrm{~A}$ high satisfaction level was also reported while using the platform as it was convenient and engaging. ${ }^{36}$ However, not all studies reported that digitalised interventions using information technology are effective or have significant benefits among women with GDM. Moreover, no significant blood glucose control improvement, and no significant differences were seen in other maternal and neonatal outcomes. ${ }^{35}$ To date the evidence is not strong enough to conclude that digital DPIs are more effective than standard DPIs for post-GDM women. ${ }^{36}$ This may be due to the need for multiple potential active elements interacting with each other in an effective mobile app. These included having the mobile apps in local language, ensuring culturally sensitive content of appropriate health advice about dietary habits and physical activities, providing motivational support and having user-friendly interfaces ${ }^{37}$ to sustain longer-term effects and outcomes. ${ }^{38}$

In short, little is known about women's experiences with GDM in Malaysia especially regarding their challenges and facilitators to uptake of DPIs. ${ }^{25}$ Hence, the objectives of this study are to:

- Explore the perceptions and factors that influence the use of existing DPIs including a digitalised DPIs (through mobile apps) among women with GDM.

- Explore the perceptions and factors influencing the use of existing DPIs including a digitalised DPI as a mobile application (app) among the HCPs who manage women with GDM and a history of GDM.

- Codevelop a mobile app between the research team members, HCPs from the participating clinics and hospitals, a hired app vendor, and women with GDM that are contextualised to the local settings and integrated with local clinical practices in an experiencebased workshop.

- Evaluate the technical quality and the experience of the mobile app (content and utility) among women with GDM. See figure 1 for an overview of study objectives.

\section{Theoretical framework}

The research model is based on Capability, Opportunity, Motivation and Behaviour (COM-B) and Behaviour Change Wheel (BCW) techniques. The COM-B model states that the complex interaction between three main factors; capability, opportunity and motivation would greatly affect the process of behavioural changes later. The factors that are perceived as challenges or motivators could affect the outcome of the behavioural change to be either positive or negative. Thus, these perceived challenges and facilitators should be addressed when planning 


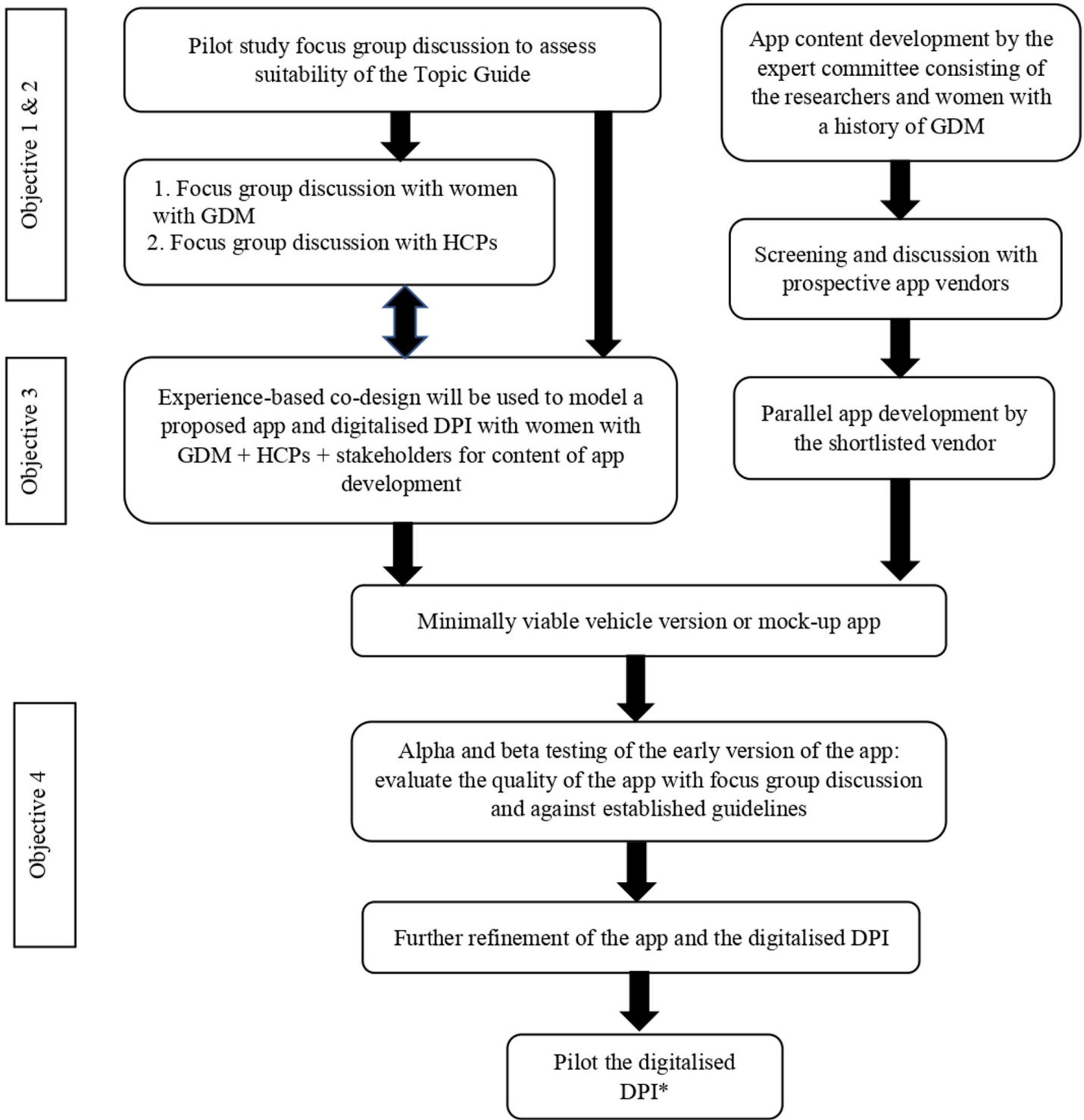

Figure 1 An overview of the study flow and how it achieves the four objectives. $\mathrm{DPI}=$ diabetes prevention intervention; HCP = healthcare providers; GDM = gestational diabetes mellitus.

an effective behavioural change technique as they can influence the three factor that create a cycle between them and behaviour change. ${ }^{39}$ The factors affecting behavioural changes will be identified in the interviews which will be integrated into the design of the BCW, and these factors will be used for the behaviour change interventions in the DPI. The BCW consist of policy categories and intervention functions, but for this DPI, we would focus primarily on intervention functions at the provider's and patient's level. ${ }^{40}$ The contents and components of the DPI will be modelled using Abraham and Michie's taxonomy of Behavioural Change Techniques ${ }^{41}$ (online supplemental file 1). This is to support moving from intentions to actions, and from actions to maintenance that include providing information on GDM and its consequences, prompting intention formation, giving guidance in challenges identification, setting up specific goal setting and self-monitoring (biofeedback such as with self-monitoring blood glucose, weight and blood pressure readings), agreeing and reviewing behavioural goals, using follow-up prompts, activating social support systems and preventing relapse. ${ }^{41}{ }^{42}$ We will also include emotional processing techniques such as ventilation, reflection and adjustment to reduce distressing experience of GDM which could lead to avoidance in making behaviour change. ${ }^{20}$

\section{METHODS AND ANALYSIS}

In planning for the context of the mobile app we will use the DoTTI development framework ${ }^{43}$ which has four phases of development (1) design and development; (2) testing early iterations; (3) testing for effectiveness; (4) integration and implementation. This protocol includes up to phase 2 of the DoTTI framework to achieve the objectives of the study. The other parts of phase 2 (the pilot testing) and subsequent phases will be described in another trial protocol. 
The three stages of assessing needs, content, interface design and development in the first phase will be conducted concurrently and iteratively. The needs assessment of the target population, women with GDM and history of GDM, and the HCPs who treat these women will be identified through interviews. Content development and consensus of the app content involve inputs from the expert committee that consist of research team members, HCPs from the participating sites of various specialties including endocrinology specialists, obstetrics and gynaecology specialists, family medicine specialists, rehabilitation medicine specialist, dietitians and the future target users. The contents of the app will be verified by these specialists to ensure that it is based on the established clinical practice guidelines and latest evidence from research. The app user interfaces will be developed by a hired app developer, and this will happen iteratively with regular sprint demonstrations and input from the research team members.

Testing early iterations of the app will involve alpha and beta testing on the functions and usability of the app. Alpha testing involves the testing of the app's interfaces such as the presentation (multimedia, wordings) and the test is usually carried out among the research team members and the app developer. The beta testing, on the other hand, will involve HCPs, women with GDM and women with a history of GDM. This will involve testing a minimally viable vehicle version of the app (the mock-up app with full functionalities). The outcome of the alpha and beta testing will indicate the aspects in the app that need further improvements. ${ }^{43}$

\section{Settings}

The study will be conducted among three public health clinics, one government hospital and one university teaching hospital for a duration of 6-12 months. These clinics and hospitals are located in the Petaling District and Federal Territory of Putrajaya. The Petaling district is in the heart of the state of Selangor with a population of 1.8 million. Almost half of the population in this district is of Malay ethnicity. ${ }^{44}$ Selangor has the highest prevalence of diabetes mellitus $(14.37 \%),{ }^{45}$ and almost one-tenth of the deliveries in 2017 were born to mothers with GDM. ${ }^{46}$ These health clinics are chosen because the study targets both urban and suburban population within the district. Putrajaya is the third federal territory of Malaysia and an administrative capital of Malaysia. Here, the majority of the population are Malays. The Putrajaya hospital and health clinic represents the urban population, which consists of a mixture of middle-income to high-income people with $90 \%$ of them are government employees. This population has the highest rate of obesity ${ }^{47}$ which is one of the risk factors for developing GDM. The majority of the population in the Petaling district and Putrajaya are of Malay ethnicity, followed by Chinese, Indians and other minorities, and this reflects the Malaysian diverse population as a whole.
The selected clinics maintain GDM registry formed by the Ministry of Health Malaysia since 2016. It includes information such as age, parity, last menstrual period, estimated due date, premature ovarian ageing, date of delivery, dates for and taken OGTT (oral glucose tolerance test) after delivery and the result of the OGTT. The GDM registry is monitored by a dedicated team (family medicine specialist and a nurse) to ensure all data are accurately and completely entered.

The clinics have an in-house dietitian who provides dietary counselling to women from different ethnic groups stated earlier. The chosen hospitals (Putrajaya and UPM teaching hospital) have a wider range of HCPs from different specialities that manage GDM. This includes obstetricians specialising in fetomaternal care and endocrinologists who manage uncomplicated and complicated cases of GDM. Both are referral centres in their respective areas.

\section{Participants}

There will be two categories of participants: (1) women with GDM and (2) HCPs. The first category of participants are women with current diagnosis of GDM and with previous GDM within 12 months post partum. Both groups of women who have past experience receiving DPI and women who refuse DPI will be invited to be the participants. The participants will be purposively selected to ensure that they are representative of women from a variety of backgrounds including all major ethnicities (Malay, Chinese and Indian), educational status (ranging from primary school to colleges or universities) and parities (primiparas and multiparas). The sample frame is the GDM registry in the respective health clinics. HCPs are those who treat women with GDM during the antenatal or/and postnatal period. Table 1 shows the inclusion and exclusion criteria for women with GDM and HCPs.

Participants will be purposively sampled for maximum variation; primarily focusing on ethnicity and parity. The diagnosis of GDM will be in accordance with the 2017 Clinical Practice Guideline: Diabetes in Pregnancy by Ministry of Health Malaysia. ${ }^{9}$ Eligible women are identified by the investigators from the GDM registry and during clinic visits at the sites (clinic or hospital). Potential participants will be approached, screened based on the eligibility criteria (table 1) and invited to a scheduled interview at the same facility where they receive medical care. During tightened movement control order (MCO) due to the COVID-19 pandemic, this participant selection process will be done in the same manner as that with the HCP as participants. Participants who are HCPs at the sites (clinic or hospital) will be identified by key informants at the respective sites. This will include senior staff and clinicians, unit heads and those who have joined this study as the site investigators. The identified participants will then be approached by the project interviewers to further confirm eligibility and availability for either on-site or online interviews. 
Table 1 Inclusion and exclusion criteria for the study population

\begin{tabular}{|c|c|c|}
\hline & Inclusion criteria & Exclusion criteria \\
\hline $\begin{array}{l}\text { Women } \\
\text { with GDM }\end{array}$ & $\begin{array}{l}\text { Pregnant women diagnosed with GDM } \\
\text { Women with a history of GDM and within } 12 \text { months post } \\
\text { partum } \\
\text { Aged } 18 \text { years and above } \\
\text { Able to communicate in English or Malay } \\
\text { Malaysian citizen } \\
\text { Owns a smartphone (iPhone or Android) }\end{array}$ & $\begin{array}{l}\text { Pre-existing diabetes (type } 1 \text { or type 2) and overt } \\
\text { diabetes } \\
\text { Chronic diseases such as end-stage renal disease, } \\
\text { heart failure, stroke and cancer } \\
\text { Mental illnesses such as psychosis, bipolar } \\
\text { disorders or learning difficulties diagnosed by } \\
\text { physicians, on treatment and documented in the } \\
\text { medical records } \\
\text { Terminal illnesses with less than } 2 \text { years of life } \\
\text { expectancy } \\
\text { Women who are not able to communicate verbally } \\
\text { Multiple pregnancies with two fetuses and above }\end{array}$ \\
\hline HCPs & $\begin{array}{l}\text { Medical officers, family medicine specialists, pharmacist, nurses } \\
\text { and dietitians who are providing direct clinical care to women } \\
\text { with GDM or a history of GDM at the participating public health } \\
\text { clinics OR } \\
\text { Endocrinologists, obstetricians and midwives working in } \\
\text { secondary care at the participating hospital AND } \\
\text { HCPs who have been in clinical service for more than } 1 \text { year in } \\
\text { their respective facility }\end{array}$ & $\begin{array}{l}\text { Non-permanent or temporary staff including the } \\
\text { non-Malaysian healthcare providers } \\
\text { House officers } \\
\text { HCPs qualified less than } 6 \text { months ago }\end{array}$ \\
\hline
\end{tabular}

GDM, gestational diabetes mellitus; HCP, healthcare provider.

The estimated sample size is 50-60 patients and 30-40 HCPs or until data saturation has been achieved. Data saturation will be reached when the team finds no further information, codes or themes emerging from the data. This will be achieved through regular cross checking of the data and discussions among the team. The estimated numbers reflect two types of focus groups, women with GDM and HCPs at the three public health clinics and the two hospitals, with 10-20 patients and about 10 HCPs from each participating clinic and hospital.

\section{Patient and public involvement}

Women with a history of GDM will be involved in deciding the content for the DPI and app. Their opinion is sought on the best delivery form for the educational content and all the functionalities in the app. Throughout this study, volunteers consisting of women with GDM and their partners, HCPs at the hospital and health clinics will be engaged to provide a contextual and personal experience with GDM and care process to model the best approach for the intervention.

\section{Phase 1: design and development}

Needs assessment

To achieve the first two objectives, a qualitative study identifying the needs, facilitators, challenges and perception of the participants on a digital DPI will run concurrently with a systematic review. A systematic review of process evaluations of interventions for the prevention of T2D will be conducted by different researchers within the team to map the features of successful apps for women with GDM. The researchers will attend joint meetings and discuss the findings regularly.

In every focus group, the women with GDM and HCPs will be provided with the context. A topic guide will consist of open-ended questions to capture the women's experience in receiving information and care as well as other factors that can affect their decisions whether or not to take up DPIs or make lifestyle changes during antenatal and postnatal period. The questions will also delve into aspects related to competing roles and responsibilities, guilt feeling, cultural and religious beliefs about diet, social pressures, economic consideration, and suggestions for a mobile app that may assist in the development of a healthy lifestyle. Topic guides for HCPs will include their experience in managing women with GDM and attempts to support these women in making lifestyle changes, identifying challenges and facilitators that influence the implementation of DPIs at healthcare facilities, making suggestions for the content of the app and improving its uptake (see online supplemental file 2). Subsequently, the findings of the mentioned factors will help to further inform the topic guide on the uptake of DPIs.

\section{Focus groups and in-depth interviews}

As a qualitative approach to data collection, focus groups and in-depths interviews will be conducted by trained researchers. Prior to the focus group, each participant will complete a short survey on the sociodemographic information such as age and experiences in using DPIs in the past. For the HCPs as participants, data will be collected based on their highest level of education and duration of clinical experience in managing GDM. The focus groups will have 5-8 participants, and the outputs are recorded in the forms of audio recordings as well as note taking by both the interviewer and note taker. All interviews may be video-taped if consented or conducted online. Both the focus groups and interviews are expected to last around $45-90 \mathrm{~min}$. The audio and video recordings 
are for analysis and will not be copied/sent to any other individual or used for any other purposes.

Face-to-face interviews may be needed if there is information gathered that needs further clarification or if participants are unable to attend a focus group discussion (FGD). This is to ensure the validity of the data. The discussion will be facilitated by a topic guide that is available in both English and Malay language. It will also be piloted on groups of women with GDM and HCPs and reviewed by the research team prior to its use. During the interview and FGD, reflexivity process will be undertaken constantly. This involves the researchers' awareness of how they affect the research. For example, how researchers articulate the social and cultural differences at the place of the research and in the context of the research will be taken into consideration. In this process, constantly revising comprehension of the situation within the research activities would bring a different understanding to it and help to increase the trustworthiness of the data. Bracketing is a process that is undertaken from the beginning of the research which allows the researchers to identify and acknowledge assumptions regarding the phenomena based on theoretical assumptions as well as the background knowledge about the research. During the interviews, discussion will be conducted using the language preferred by the participants (English, Malay or mixed). Data collection workflow can be seen in figure 2.

Should the Movement Restriction Order (MCO) be prolonged in the aftermath of the COVID-19 pandemic, all standard procedures for data collection may be conducted online using reliable and secure software/ application. Online procedures will be explained and pilot-tested with the participants before data collection begins.

\section{Content consensus}

The content of the app is discussed among the expert committee members mentioned above. Each subcontent of the app is led by small groups of people headed by an expert in the field. For example, an obstetrician, a gynaecologist and a family medicine specialist are responsible in collating the content for general information about GDM. Information on medical therapies for GDM will be managed by an endocrinologist and a family medicine specialist while dietitians are responsible for the dietary content. Content on exercises and physical activity is organised by a rehabilitation medicine physician; content on mental health is managed by health psychologists and a family medicine specialist. This content development is facilitated through two 1-day sessions for discussion and review among all the members including the app developer and women with a history of GDM. Additionally, the initial draft of the app content is shared with all Malaysia and UK research team members. The content under development will be consistently checked for compliance with clinical practice guidelines and the latest published evidence.

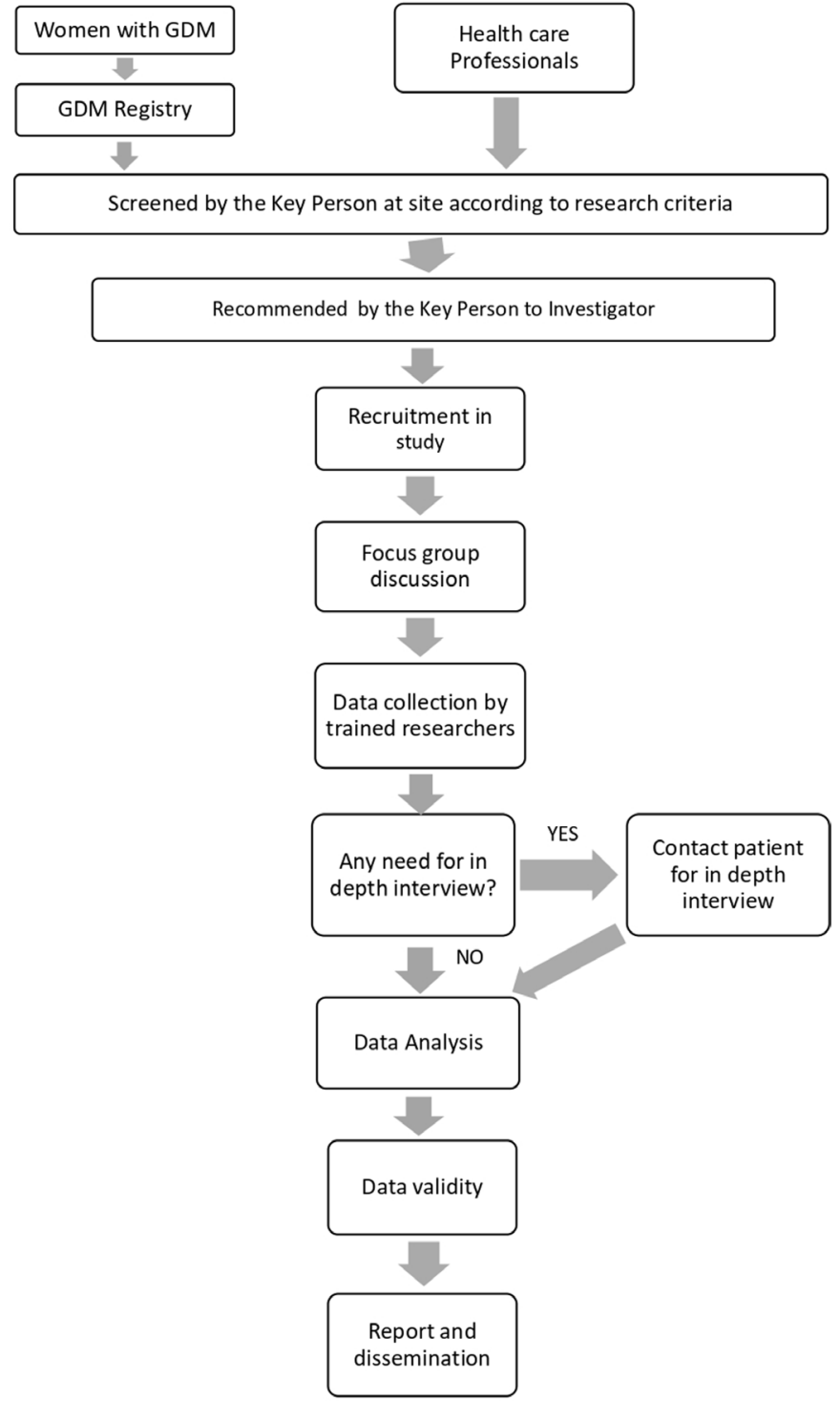

Figure 2 Recruitment and data collection process. GDM, gestational diabetes mellitus.

\section{User interface}

It is important that the developed content could be extracted by the users easily. Using results from the qualitative study and a theory-based evaluation framework (that combines factors such as credibility, information, engagement, usability and integration $)^{48}$ the app developer will design an app that is not only informative but also interesting. It is important that the presentation and interface are smooth, and that they could capture users' attention. It has been shown that the top five reasons for a user to stop using the app is related to the usability of the app such as application/system freeze or crashes, slow responsiveness, high battery consumption and within the app advertisements. ${ }^{49}$

\section{Phase 2: testing early iterations}

Alpha and beta testing

Following the production of the first minimally viable vehicle version of the app (the mock-up), we will conduct 
the alpha and beta testing for the app (Objective 5). Alpha testing will be conducted by the project team members to check for technical issues such as incorrect or broken links, misspelt words and multimedia related issues. The app is expected to deliver advice and information on healthy lifestyles using a curriculum similar to the Diabetes Prevention Programme ${ }^{13}$ online format. However, it will be culturally adapted for the health needs of GDM and post-GDM mothers in Malaysia. Adaptations will be made to fit suit the target users background, such as using the Malay language as a means of instructions and incorporating local menus and delicacies. Other suggestions that derive from the qualitative study will be included. A group webchat function may be made available where members in each group can give feedback and receive related to their progress. This support system will be facilitated by a dietitian and a physiotherapist to ensure legit healthful advice is being shared and emotional support is moderated. In case of uncertain symptoms, medical complaints or a possible adverse medical event or emergency, the participants will be advised to seek an urgent consultation with their HCPs at the health clinics and not to rely on the advice in the app.

The app will emphases on

- Usability: focusing on the quality of the interaction between the user and the technology.

- Feasibility: ensuring contextual readiness, ranging from human resource capacity to the technical ecosystem (eg, connectivity, electrical grid stability, mobile phone access) is well received. Ultimately this app aims to optimise the delivery of clinical practices by multidisciplinary HCPs.

After passing the alpha testing, we will conduct beta testing with the selected samples of HCPs $(n=15)$ and women with GDM in different trimesters $(n=15)$ and post-GDM within 12 months $(\mathrm{n}=15)$ to identify any additional issues. Partners or spouses and significant family members of the women will also be invited to use the app and to give insight into the usability of the app, of ways it may help to drive the healthy lifestyle changes in the families and the women. ${ }^{50}$ The participants will be invited to a briefing session about the app prior to testing its usability. This process will be conducted over a period of 4 weeks where participants will be encouraged to record their personal experiences in a diary that will be provided by the research team. The participants' feedbacks during the briefing will also be audio and video recorded, if consented. At the end of the 4-week, a half day workshop will be conducted to assess the participants experience with the mock-up app. This includes evaluating the mock-up app as a digitalised DPI on properties that are intrinsic (inward) to the app by using a semi structured topic guide. The participants will be in a focus group or/and in-depth one-to-one interview. The focus group and in-depth interviews will explore the thoughts, feelings, perceptions, behaviours and attitudes of the users towards the usability of the app using the System Usability Scale ${ }^{51}$ and the Mobile App Rating Scale (see online supplemental file 3$)^{5253}$ as topic guides. Additionally, an evaluation will also be conducted on metrics that are extrinsic (outward) to the app which encompass aspects that indicate better glycaemic control, healthy lifestyle adherence and psychological well-being. Ideally, the intention is to see whether changes in these extrinsic metrics are associated with the use of the app. The metrics of glycaemic control will be based on the homebased antenatal and health records as well as healthy lifestyle. Psychological well-being on the other hand, will be included as one of the topics to discuss during the interview. For this purpose, the validated Malay version of the Brief Illness Perception Questionnaire will be used as a topic guide ${ }^{54}$ Essentially, the aim of the evaluation of the metrics is to observe whether changes in these extrinsic metrics are influenced by the use of the app.

\section{Data analysis plan}

All data from the focus groups and in-depth interviews, will be transcribed verbatim and managed using qualitative data analysis software (NVivo and Atlas.ti). ${ }^{55}$ The data recorded in Malay will be translated into English to cater to collaborators and analysts who are only proficient in English. The analysis will be done by the Malaysia and British research team (on verbatim transcriptions) and will focus on differences and similarities in the aspects of care, culture and current issues as well as challenges in uptake of DPIs and the expectations of the DPIs. A separate analysis using the same approach will be conducted with the interview data from the HCPs. Framework analysis will be used to identify a thematic framework which is also guided by the behavioural change techniques model. ${ }^{56-59}$ The transcriptions will be coded in which recurring codes will establish the themes that represent the data. It is important to note that the transcriptions will have to be analysed multiple times in an ongoing manner in order to understand the true meaning that emerges from the data. Furthermore, to increase the reliability of the analysis, the data will be checked by the research team members, and this process will begin as early as the initial coding stage. Members' check will increase the trustworthiness of the data. ${ }^{58}$ Member's check will be done as early as the initial coding stage between the research team and participant involved in the interviews. Before the process of coding begins, the transcriptions will be returned to the participants to check for accuracy of the data. Findings generated from the analysis will be uploaded onto a parallel app development.

In order to enhance the credibility of this study, triangulation method will comprise interviews and focus group from both HCPs and women with GDM and views from different categories of HCPs and sociodemographic: parity and ethnicity (patients) who will give different aspects of the phenomenon, and multiple backgrounds (data triangulation) will be employed. Furthermore, employing multiple investigators for the data analysis (investigator triangulation) and data comparison (triangulating analysts) will increase the rigour of the study. 
The data gathered from the interviews will also be fed back to the participants to get their validation (respondent validation). Their feedback on the preliminary findings will be taken into account. Finally, researchers will reflect on any biases or assumptions that could have been developed (reflexivity) and the discussion of raw data, interpretations and conclusions with peers ("peer debriefing') will also be employed.

\section{DISCUSSION}

As more mothers seek guidance, tips and advice from the internet (websites, mobile apps), rather than the HCPs ${ }^{60}$ HCPs must be ready to suggest reliable and credible sources for their references. A digital application such as Mobile Health (mHealth) app can give users convenient access to important and relevant health information. This information could personalise their care and empower them to a healthier lifestyle. ${ }^{6162}$ Therefore, the findings of this study can provide insights on the experiences and perspectives of women diagnosed with GDM, about DPIs and understand their needs for using an app that supports self-management. Combining the HCPs' experiences of doing DPIs to manage GDM and the input on the usability and functionality of an app for women with GDM (present and past) the integration of the app during clinical consultation and participation from the target end-users is expected to be improved. The outcome from this study will provide rich information on what both patient and HCPs seek for building an effective and engaging digitalised DPI. By incorporating the aspects of digital use, effective DPI strategies, and elements of local context, we hope to develop an intervention that can facilitate mothers to consistently observe healthy behaviour changes as well as for the HCPs to effectively perform DPI which could potentially reduce the development of T2D in the future.

\section{ETHICS AND DISSEMINATION \\ Ethical consideration}

Written informed consent using either Malay or English will be obtained from all participants before undertaking any study procedures. Besides, informed written consent will also be obtained before the interview in which the permission is sought for audio recording the sessions, transcribing the interview data for analysis and dissemination the findings. The original research proposal was assessed and approved by MRC UK reviewers. Furthermore, this subproject has been submitted to the Malaysian Ministry of Health's Medical Research and Ethics Committee (MREC) and approval will be granted soon after the proposal is reviewed and corrections on technical issues are addressed.

\section{Privacy and confidentiality}

The participant's name will be identified using an identification number for the record of this study and for easy recognition on the consent form. For the purpose of data analysis, pseudonyms will be used to protect the identity of the participants. All data will be entered into a protected computer, and it is accessible only to the research team members. On the completion of the study, data on the computer will be copied to CDs, and the data in the computer will be erased. CDs and any hardcopy of data (including the consent forms) will be safeguarded in a locked cabinet of the principal investigator's room and be kept for a minimum of 10 years after the completion of the study.

\section{Publication policy}

No personal information will be disclosed, and participants will not be identified when the findings of the research are published. If the names and details of the patients need to be disclosed, a written expressed consent will be obtained prior to presentation and publication.

\section{Dissemination plan}

The results from this study will be published in peerreviewed scientific journals, presented in scientific conferences, and reported and shared with the local health stakeholders. Furthermore, we have also made plans to extend the use of the app in the local settings. For this purpose, we have engaged HCPs from the Ministry of Health and groups of women with a history of GDM during the content development, alpha and beta testing. Generally, the app is aimed to be used by the patients and family members, and to be a tool that helps physicians at various healthcare facilities exchange information with the patients. This app is also helpful in sharing information gathered during consultations between patients and other HCPs including nurses, midwives, dietitians, nutritionists and pharmacists. The app is currently being developed for different operating systems so that it will be available on Google Play Store and Apple Play. We have occasionally introduced the project on family physicians' social media account to generate anticipation. If the feasibility randomised controlled trial shows promising results, the project team will be working toward a full RCT to generate a more robust evidence and better prepare the app for a wider user groups and implementation.

\section{Author affiliations}

${ }^{1}$ Family Medicine, Universiti Putra Malaysia Fakulti Perubatan dan Sains Kesihatan, Serdang, Malaysia

${ }^{2}$ Faculty of Epidemiology and Population Health, London School of Hygiene and Tropical Medicine, London, UK

${ }^{3}$ Primary and Intermediate Care Section, King's College London, London, UK

${ }^{4}$ Biostatistics and Health Informatics, Institute of Psychiatry, King's College London, London, UK

${ }^{5}$ Women and Children's Health, Faculty of Life Sciences and Medicine, King's College London, London, UK

${ }^{6}$ Research Center for Optimal Health, University of Westminster, London, UK

${ }^{7}$ Nutrition and Dietetics, University Putra Malaysia, Serdang, Malaysia

${ }^{8}$ Obstetrics and Gynaecology, Universiti Putra Malaysia Fakulti Perubatan dan Sains Kesihatan, Serdang, Malaysia

${ }^{9}$ Rehabilitation, Universiti Putra Malaysia, Serdang, Malaysia

${ }^{10}$ Clinical Research Unit, Universiti Putra Malaysia Hospital Pengajar Universiti Putra Malaysia, Serdang, Malaysia 
${ }^{11}$ Institute of Psychiatry, King's College London, London, UK

\section{Twitter Boon How Chew @chewboonhow}

Acknowledgements We would like to thank the Ministry of Education Malaysia through the MyPAiR (Malaysia Partnership \& Alliances in Research) programme and the UK's Medical Research Council that have jointly created the MyPAiR-Noncommunicable Disease Year 2019 research grant for providing the funding for this study which is a part of the MalaYsian GestatiOnal Diabetes and Prevention of DiabetES Study (MYGODDESS). We would like to thank MYGODDESS Project team members and collaborators include MSc student Ms Pamela Phui Har Yap, Dr Ziti Akthar Supian at Seri Kembangan Health Clinic, Dr Haslinda Hassan at Puchong Health Clinic, Dr Fuziah Paimin at Putrajaya Presint 9 Health Clinic, Dr Nurain Mohd. Noor and Dr Wan Ahmad Hazim Wan Ghazali from Medical and Obstetrics and Gynaecology departments in Hospital Putrajaya, respectively.

Contributors All authors conceived the study from the beginning. $\mathrm{KI}$ and $\mathrm{BHC}$ designed the whole study. NHMS drafted the initial draft assisted by IZI, FH and IPN. All authors (NHMS, IZI, FH, AF, SMC, HA, KG, HM, NG, BNMY, NIB, MSS, CA, IIMS, $\mathrm{BHC}, \mathrm{KI})$ critically revised the study protocol and approved the final manuscript for publication. $\mathrm{KI}$ and BHC are the guarantors of the study. MYGODDESS Project team members and collaborators include MSc student Ms Pamela Phui Har Yap, Dr Ziti Akthar Supian at Seri Kembangan Health Clinic, Dr Haslinda Hassan at Puchong Health Clinic, Dr Fuziah Paimin at Putrajaya Presint 9 Health Clinic, Dr Nurain Mohd. Noor and Dr Wan Ahmad Hazim Wan Ghazali from Medical and Obstetrics and Gynaecology departments in Hospital Putrajaya, respectively. They are the research site investigators and will function as key informants to the study in recruiting participants and facilitating logistics arrangement at the research sites.

Funding This work is supported by Malaysian Ministry of Higher Education's MYPAIR Grant UK-Malaysia: Joint Partnership Call on Non-Communicable Diseases (JPT.S(BPKI)2000/011/06/05 (27); and the Medical Research Council UK (MR/ T018240/1) https://cuttly/Tl1Aljb.

Competing interests None declared.

Patient consent for publication Not required.

Provenance and peer review Not commissioned; externally peer reviewed.

Supplemental material This content has been supplied by the author(s). It has not been vetted by BMJ Publishing Group Limited (BMJ) and may not have been peer-reviewed. Any opinions or recommendations discussed are solely those of the author(s) and are not endorsed by BMJ. BMJ disclaims all liability and responsibility arising from any reliance placed on the content. Where the content includes any translated material, BMJ does not warrant the accuracy and reliability of the translations (including but not limited to local regulations, clinical guidelines, terminology, drug names and drug dosages), and is not responsible for any error and/or omissions arising from translation and adaptation or otherwise.

Open access This is an open access article distributed in accordance with the Creative Commons Attribution Non Commercial (CC BY-NC 4.0) license, which permits others to distribute, remix, adapt, build upon this work non-commercially, and license their derivative works on different terms, provided the original work is properly cited, appropriate credit is given, any changes made indicated, and the use is non-commercial. See: http://creativecommons.org/licenses/by-nc/4.0/.

\section{ORCID iDs}

Nur Hafizah Mahamad Sobri http://orcid.org/0000-0003-0857-4407

Faezah Hassan http://orcid.org/0000-0002-0891-3934

Angus Forbes http://orcid.org/0000-0003-3331-755X

Siew Mooi Ching http://orcid.org/0000-0003-0505-019X

Boon How Chew http://orcid.org/0000-0002-8627-6248

\section{REFERENCES}

1 Cho NH, Shaw JE, Karuranga S, et al. IDF diabetes atlas: global estimates of diabetes prevalence for 2017 and projections for 2045. Diabetes Res Clin Pract 2018;138:271-81.

2 Nguyen CL, Pham NM, Binns CW, et al. Prevalence of gestational diabetes mellitus in eastern and southeastern Asia: a systematic review and meta-analysis. J Diabetes Res 2018;2018:6536974 doi:10.1155/2018/6536974

3 Jai AN, Kassim ABM, Samad AA. National Health and Morbidity survey 2016: maternal and child health $(\mathrm{MCH}$. . Kementerian Kesihatan Malaysia, 2016: 2. 1-276. http://www.iku.gov.my/images/
IKU/Document/REPORT/2016/NHMS2016ReportVolumell-Maternal ChildHealthFindingsv2.pdf

4 Kampmann U, Madsen LR, Skajaa GO, et al. Gestational diabetes: a clinical update. World J Diabetes 2015;6:1065.

5 Coustan DR, Jovanovic L. Gestational diabetes mellitus: glycemic control and maternal prognosis. UpToDate, 2015: 1-25. https://www. uptodate.com/contents/gestational-diabetes-mellitus-glycemiccontrol-and-maternal-prognosis?search=metformin-regular-drug\& source=search_result\&selectedTitle=8 150\&usage_type=default\& display_rank $=8$

6 Kim C, Newton KM. Knopp Rh. gestational diabetes and the incidence of type 2 diabetes. Diabetes Care 2002;25:1862-8.

7 , Metzger BE, Lowe LP, et al, HAPO Study Cooperative Research Group. Hyperglycemia and adverse pregnancy outcomes. N Engl J Med 2008;358:1991-2002.

8 Aroda VR, Christophi CA, Edelstein SL, et al. The effect of lifestyle intervention and metformin on preventing or delaying diabetes among women with and without gestational diabetes: the diabetes prevention program outcomes study 10-year follow-up. J Clin Endocrinol Metab 2015;100:1646-53 https://doi.org/10.1210/jc. 2014-3761

9 Ministry of Health Malaysia. Clinical Practice Guideline Diabetes. In: Pregnancy (MOH/P/PAK/353.17(GU)), 2017. https://www.moh.gov. $\mathrm{my} / \mathrm{moh} /$ resources/Penerbitan/CPG/Endocrine/1a.pdf

10 Sugiyama T. Management of gestational diabetes mellitus. Jpn Med Assoc J 2011;54:293-300.

11 Navneet M, Savitha P, Seshiah V. Medical nutrition therapy in gestational diabetes mellitus, 2015. Available: https://www. researchgate.net/publication/274698499 Medical Nutrition Therapy_in_Gestational_Diabetes_Mellitus [Accessed Mar 2015].

12 Reader D, Splett P, Gunderson EP, et al. Impact of gestational diabetes mellitus nutrition practice guidelines implemented by registered dietitians on pregnancy outcomes. J Am Diet Assoc 2006;106:1426-33.

13 The diabetes prevention program outcomes study 10-year follow-up. $J$ Clin Endocrinol Metab 2015;100:1646-53.

$14 \mathrm{Xu}$ T, He Y, Dainelli L, et al. Healthcare interventions for the prevention and control of gestational diabetes mellitus in China: a scoping review. BMC Pregnancy Childbirth 2017;17:1-10.

15 Knowler WC, Barrett-Connor E, Fowler SE, et al. Reduction in the incidence of type 2 diabetes with lifestyle intervention or metformin. N Engl J Med 2002;346:393-403.

16 Diabetes Prevention Program Research Group. Long-Term effects of lifestyle intervention or metformin on diabetes development and microvascular complications over 15-year follow-up: the diabetes prevention program outcomes study. Lancet Diabetes Endocrinol 2015;3:866-75

17 Goveia P, Cañon-Montañez W, Santos DdeP, et al. Lifestyle intervention for the prevention of diabetes in women with previous gestational diabetes mellitus: a systematic review and meta-analysis. Front Endocrinol 2018;9:583.

18 Hui AL, Sevenhuysen G, Harvey D, et al. Barriers and coping strategies of women with gestational diabetes to follow dietary advice. Women Birth 2014:27:292-7.

19 Hui AL, Sevenhuysen G, Harvey D, et al. Food choice decisionmaking by women with gestational diabetes. Can J Diabetes 2014;38:26-31.

20 Parsons J, Sparrow K, Ismail K. Experiences of gestational diabetes and gestational diabetes care: a focus group and interview study. BMC Pregnancy and Childbirth 2018;18:1-12.

21 Parsons J, Ismail K, Amiel S, et al. Perceptions among women with gestational diabetes. Qual Health Res 2014;24:575-85.

22 Martis R, Brown J, Crowther CA. Views and experiences of new Zealand women with gestational diabetes in achieving glycaemic control targets: the views study. J Diabetes Res 2017;2017:2190812

23 Nielsen KK, Kapur A, Damm P, et al. From screening to postpartum follow-up - the determinants and barriers for gestational diabetes mellitus (GDM) services, a systematic review. BMC Pregnancy Childbirth 2014;14:41.

24 Lucas C, Charlton KE, Yeatman H. Nutrition advice during pregnancy: do women receive it and can health professionals provide it? Matern Child Health J 2014;18:2465-78.

25 Nur Suraiya AHS, Zahara A, Nazlena MA. Perspectives of healthcare professionals and patients on management of gestational diabetes mellitus: a qualitative study in Negeri Sembilan, Malaysia. Malays $J$ Nutr 2016:21:393-9.

26 Wilkinson SA, McCray S, Beckmann M, et al. Barriers and enablers to translating gestational diabetes guidelines into practice. Practical Diabetes 2014:31:67-72. 
27 Oza-Frank R, Ko JY, Wapner A, et al. Improving care for women with a history of gestational diabetes: a provider perspective. Matern Child Health J 2014;18:1683-90.

28 Hewage SS, Singh SR, Chi C, et al. Health care providers' perceptions of responsibilities and resources to reduce type 2 diabetes risk after gestational diabetes mellitus. Clin Diabetes 2018;36:160-7.

29 El-Gayar O, Timsina P, Nawar N, et al. A systematic review of it for diabetes self-management: are we there yet? Int J Med Inform 2013;82:637-52.

30 Immanuel J, Simmons D. Apps and the woman with gestational diabetes mellitus. Diabetes Care 2021;44:313-5.

31 Chen Q, Carbone ET, Functionality CET. Functionality, implementation, impact, and the role of health literacy in mobile phone Apps for gestational diabetes: Scoping review. JMIR Diabetes 2017;2:e25-13.

32 World Health Orgnaization. Monitoring and evaluating digital health interventions: a practical guide to conducting research and assessment, 2016. Available: https://www.who.int/ reproductivehealth/publications/mhealth/digital-health-interventions/ en/ [Accessed Mar 2020].

33 Mackillop L, Hirst JE, Bartlett KJ, et al. Comparing the efficacy of a mobile phone-based blood glucose management system with standard clinic care in women with gestational diabetes: randomized controlled trial. JMIR Mhealth Uhealth 2018;6:e71.

34 Miremberg $\mathrm{H}$, Ben-Ari T, Betzer T, et al. The impact of a daily smartphone-based feedback system among women with gestational diabetes on compliance, glycemic control, satisfaction, and pregnancy outcome: a randomized controlled trial. Am J Obstet Gynecol 2018;218:453.e1-453.e7.

35 Ming W-K, Mackillop LH, Farmer AJ, et al. Telemedicine technologies for diabetes in pregnancy: a systematic review and meta-analysis. $J$ Med Internet Res 2016;18:e290-12.

36 Yew TW, Chi C, Chan S-Y, et al. A randomized controlled trial to evaluate the effects of a smartphone Application-Based lifestyle coaching program on gestational weight gain, glycemic control, and maternal and neonatal outcomes in women with gestational diabetes mellitus: the SMART-GDM study. Diabetes Care 2021;44:456-63.

37 Alnasser AA, Alkhalifa AS, Sathiaseelan A, et al. What overweight women want from a weight loss APP: a qualitative study on Arabic women. JMIR Mhealth Uhealth 2015;3:e41.

38 Lunde $\mathrm{P}$, Nilsson BB, Bergland A, et al. Feasibility of a mobile phone app to promote adherence to a heart-healthy lifestyle: single-arm study. JMIR Form Res 2019;3:e12679-12.

39 McDonagh LK, Saunders JM, Cassell J, et al. Application of the COM-B model to barriers and facilitators to Chlamydia testing in general practice for young people and primary care practitioners: a systematic review. Implement Sci 2018;13:130.

40 Michie S, van Stralen MM, West R. The behaviour change wheel: a new method for characterising and designing behaviour change interventions. Implement Sci 2011;6:42.

41 Abraham C, Michie S. A taxonomy of behavior change techniques used in interventions. Health Psychol 2008;27:379-87.

42 Baptiste-Roberts K, Barone BB, Gary TL, et al. Risk factors for type 2 diabetes among women with gestational diabetes: a systematic review. Am J Med 2009;122:207-14.

43 Department of Statistics Malaysia. Population distribution and basic demographic characteristic report 2010 (Vol. 2012, issue 14 October)., 2010. Available: http://www.statistics.gov.my/portal/index. php?option=com_content\&view=article\&id=1215\%3Apopulationdistribution-and-basic-demographic-characteristic-reportpopulation-and-housing-census-malaysia-2010-updated-2972011\& catid $=130 \% 3$ Apopulation-distribution-and
44 Smits R, Bryant J, Sanson-Fisher R, et al. Tailored and integrated web-based tools for improving psychosocial outcomes of cancer patients: the DoTTI development framework. J Med Internet Res 2014;16:e76-13.

45 Chandran A, Mohd Nazri Abdullah FA. National diabetes Registry report 2013-2019. Disease Control Division Ministry of Health Malaysia, 2019: 1-34. http://www. aafp.org/afp/2006/0915/p971. html\%5Cnhttp://search.proquest.com/docview/216298826? accountid=15115\%5Cnhttp://ndep.nih.gov/media/youth_factsheet. pdf

46 Jeganathan R, Karalasingam SD. 5th Report of National Obstetrics Registry 2016 - 2017. National Obstetrics Registry Publisher, 2020. http://www.acrm.org.my/nor/

47 Ministry of Health Malaysia, I. for P. H. National health and morbidity survey 2015 non communicable diseases, risk factors and other health problems., 2015. Available: https://www.moh.gov.my/moh/ resources/nhmsreport2015vol2.pdf

48 Tassone C, Keshavjee K, Paglialonga A, et al. Evaluation of mobile apps for treatment of patients at risk of developing gestational diabetes. Health Informatics J 2020;26:1983-94.

$49 \mathrm{~N}$ Inukollu V, Keshamon DD, Kang T, et al. Factors Influncing quality of mobile Apps: role of mobile APP development life cycle. IJSEA 2014;5:15-34.

50 McManus R, Miller D, Mottola M, et al. Translating healthy living messages to postpartum women and their partners after gestational diabetes (GDM): body habitus, A1c, lifestyle habits, and program engagement results from the families Defeating diabetes (FDD) randomized trial. Am J Health Promot 2018:32:1438-46.

51 Brooke J. SUS- a quick and dirty usability scale. . Usability Evaluation in Industry, 1996: 189. 4-7.

52 Mohamad Marzuki MF, Yaacob NA, Yaacob NM. Translation, crosscultural adaptation, and validation of the Malay version of the system usability scale questionnaire for the assessment of mobile apps. JMIR Hum Factors 2018:5:1-7.

53 Stoyanov SR, Hides L, Kavanagh DJ, et al. Mobile app rating scale: a new tool for assessing the quality of health mobile apps. JMIR Mhealth Uhealth 2015;3:e27.

54 Chew B-H, Vos RC, Heijmans M, et al. Validity and reliability of a Malay version of the brief illness perception questionnaire for patients with type 2 diabetes mellitus. BMC Med Res Methodol 2017; $17: 118$.

55 Zakaria R, Hatib Musta'amal A, Amin NFM. Transcribing with ATLAS. ti. ATLAS.Ti user conference 2015 - qualitative data analysis and beyond, 2016: 1-15.

56 Gale NK, Heath G, Cameron E, et al. Using the framework method for the analysis of qualitative data in multi-disciplinary health research. BMC Med Res Methodol 2013;13:117-25.

57 Hackett A, Strickland K. Using the framework approach to analyse qualitative data: a worked example. Nurse Res 2019;26:8-13.

58 Nowell LS, Norris JM, White DE. Thematic analysis: Striving to meet the Trustworthiness criteria. Int J Qual 2017;16:1-13.

59 Smith J, Firth J. Qualitative data analysis: the framework approach Nurse Res 2011;18:52-62.

60 Sayakhot P, Carolan-Olah M. Sources of information on gestational diabetes mellitus, satisfaction with diagnostic process and information provision. BMC Pregnancy Childbirth 2016;16:1-9.

61 Kumar S, Nilsen WJ, Abernethy A, et al. Mobile health technology evaluation: the mHealth evidence workshop. Am J Prev Med 2013;45:228-36.

62 Muralidharan S, Ranjani H, Anjana RM, et al. Mobile health technology in the prevention and management of type 2 diabetes. Indian J Endocrinol Metab 2017;21:334-40. 